\title{
DEFECT RECONSTRUCTION AFTER HIP TUMOR RESECTION - OUR CLINICAL EXPERIENCE
}

doi: 10.2478/rojost-2018-0076

G.O. Muntean ${ }^{1}$, M. Nica1, T.Ș. Dumitrescu ${ }^{2}$

${ }^{1}$ Sf. Constantin Hospital, Brașov, Romania

${ }^{2}$ County Clinical Emergency Hospital Brașov, Romania

Aim. Short and mid-term analysis of postoperative results after hip vicinity tumor resection and reconstruction with salvage of the lower limb.

Material and method. Retrospective study on a number of 13 cases: 6 pelvis tumors, 3 femur tumors and 4 femur and pelvis tumors. 3 out of the total pelvis tumors were treated using custom pelvis reconstruction prosthesis, the other ones using bone graft and standard implants. The femoral tumors were treated using modular prosthesis and bone graft and osteosynthesis implants.

Results. Short-term outcome was favorable.

Conclusion. Bone defect reconstruction after hip vicinity tumor resection is a technically difficult procedure, which requires significant material resources. In terms of quality of life, the results are clearly superior compared to tumor resection with the sacrifice of the affected member.

Keywords: hip tumor reconstruction, custom hip prosthesis, modular prosthesis, bone defect reconstruction 